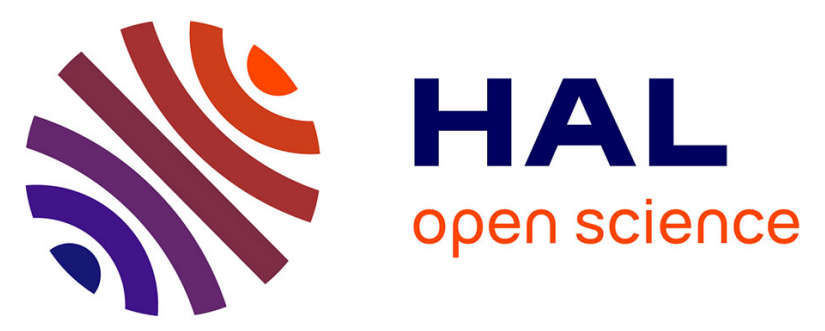

\title{
Engineering a light-matter strong coupling regime in perovskite-based plasmonic metasurface: quasi-bound state in the continuum and exceptional points
} Leran Lu, Quynh Le-Van, Lydie Ferrier, Emmanuel Drouard, Christian Seassal, Hai Son Nguyen

\section{To cite this version:}

Leran Lu, Quynh Le-Van, Lydie Ferrier, Emmanuel Drouard, Christian Seassal, et al.. Engineering a light-matter strong coupling regime in perovskite-based plasmonic metasurface: quasibound state in the continuum and exceptional points. Photonics research, 2020, 8 (12), pp.A91. 10.1364/PRJ.404743 . hal-03064608

\section{HAL Id: hal-03064608 https://hal.science/hal-03064608}

Submitted on 14 Dec 2020

HAL is a multi-disciplinary open access archive for the deposit and dissemination of scientific research documents, whether they are published or not. The documents may come from teaching and research institutions in France or abroad, or from public or private research centers.
L'archive ouverte pluridisciplinaire HAL, est destinée au dépôt et à la diffusion de documents scientifiques de niveau recherche, publiés ou non, émanant des établissements d'enseignement et de recherche français ou étrangers, des laboratoires publics ou privés. 


\title{
Engineering light-matter strong coupling regime in perovskite-based plasmonic metasurface: quasi-bound state in the continuum and exceptional points
}

\author{
LERAN LU, ${ }^{1}$ QUYNH LE-VAN ${ }^{2}{ }^{2}$ LydIE FERRIER, ${ }^{3}$ EMMANUEL \\ Drouard, ${ }^{1}$ Christian Seassal, ${ }^{1}$ And HaI Son NGuYen ${ }^{1,4},{ }^{*}$ \\ ${ }^{1}$ Université de Lyon, Institut des Nanotechnologies de Lyon, INL/CNRS, Ecole Centrale de Lyon, 36 avenue \\ Guy de Collongue, 69130 Ecully, France \\ ${ }^{2}$ College of Engineering and Computer Science, VinUniversity, Hanoi 14000, Vietnam \\ ${ }^{3}$ Université de Lyon, Institut des Nanotechnologies de Lyon, INL/CNRS, INSA-Lyon, 7 avenue Jean Capelle, \\ 69621 Villeurbanne, France \\ ${ }^{4}$ Institut Universitaire de France (IUF) \\ *hai-son.nguyen@ec-lyon.fr
}

\begin{abstract}
We present theoretically the formation of exciton-photon polaritons and excitonsurface plasmon polaritons in perovskite-based subwavelength lattice on metallic plane. It is showed that the later polaritons will be achieved as the perovskite layer is ultra thin $(<50 \mathrm{~nm})$ while the co-existence of both polaritons will dominate as the thickness of the perovskite metasurface approaches wavelength-scale. In the two cases, the lower polaritonic branches consist of dark and bright modes corresponding to infinite and finite radiative quality factor, respectively. Another salient property in this work is that it allows one to obtain exceptional points (EPs) in momentum space with a four-fold enhancement of local density of states through engineering the perovskite metasurface. Our findings show that perovskite metasurface is an attractive and rich platform to make polaritonic devices, even with the presence of lossy metallic layer.
\end{abstract}

(C) 2020 Chinese Laser Press

\section{Introduction}

Polaritons are eigenstates formed by strong interactions between the excitations of two oscillators: electromagnetic waves and matter. The energy exchange rate between two oscillators is so rapid that it exceeds the decoherent processes of each oscillator, resulting in the creation of polaritons which is evidenced by an avoided crossing in the energy-momentum curve. These quantum states, that are mixed light-matter quasi-particles, have attracted considerable interest in photonic research. Besides the traditional exciton-photon polaritons [1], the emergence of modern plasmonics has added new categories of polaritons such as exciton-plasmon polaritons $[2,3]$ and exciton-surface plasmon polaritons (ESPP) [4-8]. The ESPP is a special case when a strong coupling between the excitons of quantum emitters and surface plasmon polaritons (SPPs) occurs. The ESPP consists of electronic energy levels that are distinctive from the emitters and the SPP individually. Despite of original differences on the light side, they all possess bosonic nature and inherit the best features of their components. Specifically, the photonic/surface plasmon part offers ballistic propagation over macroscopic distances $[9,10]$, as well as optical means to generate and probe polaritons [11]. In parallel, the exciton in the matter plays the role of the active component, not only for light emission but also for nonlinearity interaction which is orders of magnitude stronger than pure photonic Kerr effect $[12,13]$. These special traits make polaritons a fascinating platform to study out-of-equilibrium Bose-Einstein condensation $[14,15]$, quantum fluid of light $[16,17]$ and topological photonics $[18,19]$. They also enable various applications with novel devices for lasing [20], integrated photonics [21,22], photovoltaics [23], 
sensing [24], photocatalysis and chemical kinetics [25, 26].

Among active materials for polaritonic devices operating at room temperature, halide perovskites - a type of semiconductors containing organic molecules, lead, and group VII element such as $(\mathrm{Cl}, \mathrm{Br}, \mathrm{I})$ - have recently emerged as an ideal candidate thanks to their excellent properties and low-cost fabrication. Unlike excitons of other room temperature active materials, perovskite excitons manifest strong nonlinear behaviors of Wannier-Mott type $[27,28]$ while exhitbiting high binding energy upto a few hundreds meV as strong as that of Frenkel excitons [29-31]. This makes them robust excitons at room temperature. Up to now, making use of perovskite platform, different groups have successfully demonstrated polariton Bose-Einstein condensation (i.e. polariton lasing) [32-39], laser cooling [40], ballistic propagation [33], exciton-surface plasmon polaritons [6,41], polaritonic microstructures [33,42-44] and lattices [36, 45-47]. However, the polaritonic devices reported in these works are optically pumped. In order to realize practical devices, metallic materials are usually introduced to inject them electrically. The use of metals will induce significant challenges, for example, a decreased quality factor of the polariton modes due to unavoidable absorptive losses of the metal. Beside the electrical properties of the metal film, we will exploit its surface plasmons coupled with excitons of the perovskite to realize the formation of exciton-surface plasmon polaritons which have also been observed in several works $[6,41]$. Lately, it has been demonstrated that perovskite-based metasurface of subwavelength periodic lattice offers an on-demand energy-momentum polaritonic dispersion [47]. While this work only discussed on the real part (i.e. energy) of polaritonic dispersion, textured metasurfaces can offer novel strategies to overcome the challenges by engineering the imaginary part (i.e. losses) via concepts such as bound states in the continuum (BIC) $[48,49]$ or exceptional points [50-53]. As a result, the metasurface approach would be a promising configuration to minimize the effect of metallic layer when designing electrically injected polaritonic devices.

In this work, we investigate theoretically the formation of exciton-photon polaritons and exciton-surface plasmon polaritons in perovskite-based lattice of subwavelength period on metallic substrate. It is showed that depending on the perovskite layer, two different regimes are obtained: solely exciton-surface plasmon polaritons for ultrathin metasurface, and the co-existence of exciton-photon polaritons and exciton-surface plasmon polaritons for metasurface of wavelength-scale thickness. In both cases, the lower polaritonic branches consist of dark and bright modes corresponding to infinite and finite radiative quality factor, respectively. Another salient property of the system is that it allows one to obtain exceptional points with an enhancement of local density of states by simple tuning of geometrical parameters of the perovskite film. In particular, the configuration sustains exciton-photon polaritons which can be designed in such a way that the nonradiative losses of the dark mode is negligible and this state becomes a quasi-BIC of high quality factor. Our findings show that perovskite metasurface is an attractive and rich platform to make polaritonic devices, even with the presence of metallic layer.

\section{Surface Plasmon Polariton (SPP) and Dielectric Guided Mode (DGM) of Per- ovskite thin film on metallic substrate}

We first investigate different guided modes formed within a perovskite thin film deposited on metallic substrate. Two types of guided modes, distinguished by field localization and polarization, are expected: i) Surface Plasmon Polariton (SPP) which is localized at the interface perovskite-metal and only exhibit TM-polarized light; ii) Dieclectric Guided Mode (DGM) which is localized within the perovskite layer and can exhibit both TE and TM-polarized light. These modes will serve as a basis to engineer photonic/plasmonic modes in the perovskite metasurface.

To model the perovskite material, we use the dielectric function of phenethylammonium lead 

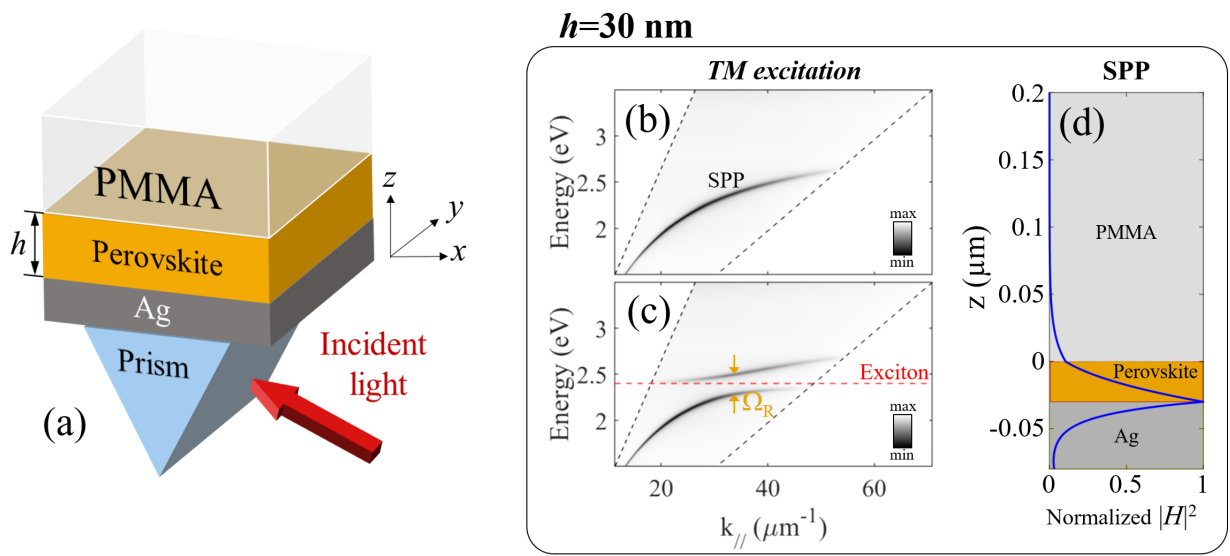

\section{$h=150 \mathrm{~nm}$}

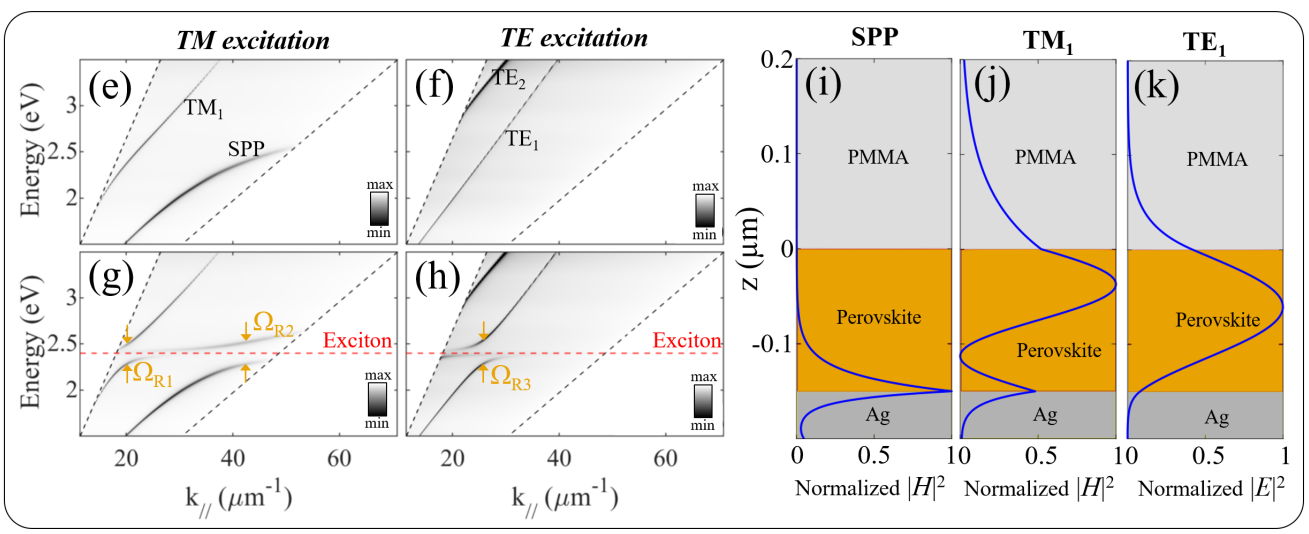

Fig. 1. (a) Sketch of the Kretschmann-Raether configuration to investigate guided modes in a stack consisting encapsulated perovskite thin film of thickness $h$ on silver substrate. (b,c,d) Results of transfer matrix calculations corresponding to the case $h=30 \mathrm{~nm}$ : (b,c) Angle-resolved reflectivity spectra in TM polarized-light of (b) passive and (c) active structures; (d) Field distribution of the SPP mode shown in (b). (e-k) Results of transfer matrix calculations corresponding to the case $h=150 \mathrm{~nm}$ : (e-h) Angle-resolved reflectivity spectra in (e,g) TM and (f,h) TE polarized-light of (e,f) passive and (g,h) active structures; (i-k) Field distribution of modes shown in (e,f).

iodide (PEPI) perovskite [i.e. $\left(\mathrm{C}_{6} \mathrm{H}_{5} \mathrm{C}_{2} \mathrm{H}_{4} \mathrm{NH}_{3}\right)_{2} \mathrm{PbI}_{4}$ ], one of the most used perovskite materials for polaritonic applications $[27,31,37,42,47]$. In this model, the excitonic resonance is described by a Lorentz oscillator, with dielectric function given by [54]:

$$
\epsilon_{\text {active }}(E)=n_{\infty}^{2}+\frac{A}{\left(E_{X}^{2}-E^{2}\right)+i \Gamma_{X} E}
$$

where $n_{\infty}=2.4$ is the refractive index of the passive material, $E_{X}=2.4 \mathrm{eV}$ is the energy of excitonic resonance, $\Gamma_{X}=0.03 \mathrm{eV}$ is its homogeneous linewidth, and $A=0.4 \mathrm{eV}^{2}$ is the constant related to its oscillator strength. In the following, when refering to the passive structure, the Lorentz oscillator is taken out and the dielectric function is simply the passive material: $\epsilon_{\text {passive }}=n_{\infty}^{2}$. The metallic substrate is made of silver with dielectric function taken from the literature [55]. The superstrate is made of Poly-methyl methacrylate (PMMA, $n=1.49$ ) to mimic 
real configurations in which perovskite layers are often encapsulated in a polymer layer to avoid the impact of moisture $[47,56]$.

Kretschmann-Raether configuration [57] is used to excite SPPs and DGMs with external light [see Fig.1.(a)]. These guided modes are evidenced as resonant dips in reflectivity spectra obtained by transfer matrix calculations with excitation in TM polarization (i.e. polarized in the xz-plane) and TE polarization (i.e. polarized in the xy-plane). The angle-resolved reflectivity spectra of passive structures are depicted in Figs.1.(b,e,f), with corresponding field distributions shown in Figs.1.(d,i,j,k), and corresponding angle-resolved spectra of active structures depicted in Figs.1.(c,g,h). These results show that depending on the perovskite thickness $h$, two different situations are obtained as discussed in the following.

If the thickness of perovskite layer is ultrathin, for example $h=30 \mathrm{~nm}$, the passive structure only sustains SPP mode [Fig.1.(b)] which is localized at the interface perovskite-silver [Fig.1.(d)]. In the active structure, the strong coupling regime between the SPP mode and exciton is distinctly evidenced by the observation of anticrossing with Rabi splitting of $\Omega_{R}=225 \mathrm{meV}$ [Fig.1.(c)].

When the optical thickness of perovskite layer exceeds half-wavelength-scale, for example $h=150 \mathrm{~nm}$, the passive structure can sustain both SPP mode and DGM modes $\left(\mathrm{TM}_{1}\right.$ in TM polarization and $\mathrm{TE}_{1}, \mathrm{TE}_{2}$ in $\mathrm{TE}$ polarization) [Figs.1.(e,f)]. Since the $\mathrm{TE}_{2}$ mode is at much higher energy than the excitonic resonance, we only focus on $\mathrm{SPP}, \mathrm{TM}_{1}$ and $\mathrm{TE}_{1}$ modes. Their field distributions are depicted in Figs.1.(i-k). We note that while mainly localized within the perovskite film, the $\mathrm{TM}_{1}$ still has a small amount localized at the interface perovskite-silver. This mode is then not purely DGM but a hybrid of SPP and DGM. In the active structures, both SPP, $\mathrm{TM}_{1}$ and $\mathrm{TE}_{1}$ undergo strong coupling with the excitonic resonance. It results in anticrossings with Rabi splittings amount to $\Omega_{R 1}=241 \mathrm{meV}, \Omega_{R 2}=207 \mathrm{meV}$ and $\Omega_{R 3}=260 \mathrm{meV}$ respectively [Figs.1.(g,h)].

\section{Bloch resonances of SPP and DGM in weakly patterned structure}

The design of our perovskite metasurface is a grating of period $a$ in the range of 250-300 nm, with filling fraction $0<f<1$. The total thickness of perovskite is $h=30$ or $150 \mathrm{~nm}$ with etching ratio $0<\eta<1$ [See Fig.2.(a)]. From the results of the previous section, we know that the penetration depth of different modes into the metallic substrate can be negligible, thus the silver substrate can be replaced by $100 \mathrm{~nm}$ of silver on silicon substrate. We note that the patterning of perovskite can be fabricated by thermal nano-imprint [58-60] or electron-beam lithography followed by a dry etching $[56,61,62]$.

Periodic patterning of perovskite layer would out-couple guided modes to the free-space via Bragg scattering: SPP and DGM becomes Bloch resonances via band-folding [63]. When the periodic modulation is strong enough (i.e. high contrast refractive-index grating), Bloch resonances of counter-propagating waves can couple and lead to gap-opening. It is straight forward that the dispersion and losses of these modes, as well as their coupling with excitons, can be strongly controlled via band-gap engineering.

To identify the guided resonances for band-gap engineering, it is important to study the weakly patterned structure $(f \sim 1$ and $\eta \ll 1)$. The dispersion of these modes are obtained by folding the dispersion of guided modes extracted from Figs.1.(b,e,f) into the first Brillouin zone. These folded dispersion are depicted in Figs.2.(b,d,e,f). The index $m= \pm 1, \pm 2$ attributed to each branch is the diffraction order corresponding to the shift of $2 m \pi / a$ in the momen- 


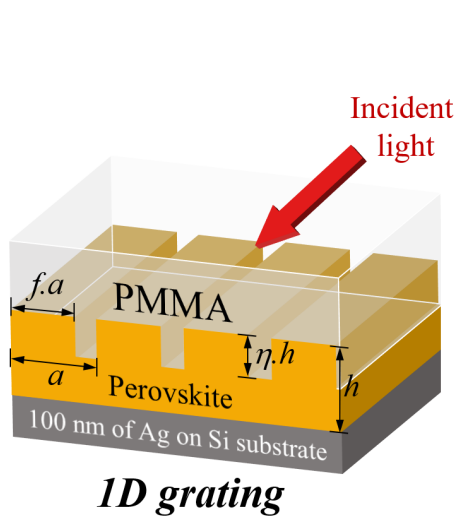

(a)
Weakly modulated $1 \mathrm{D}$ grating $(\mathrm{h}=30 \mathrm{~nm})$

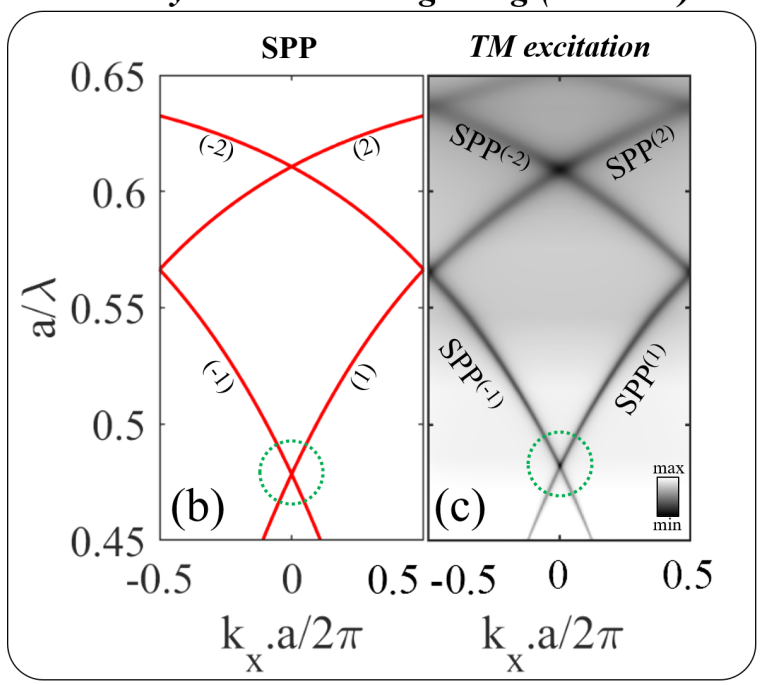

Weakly modulated 1D grating $(h=150 \mathrm{~nm})$

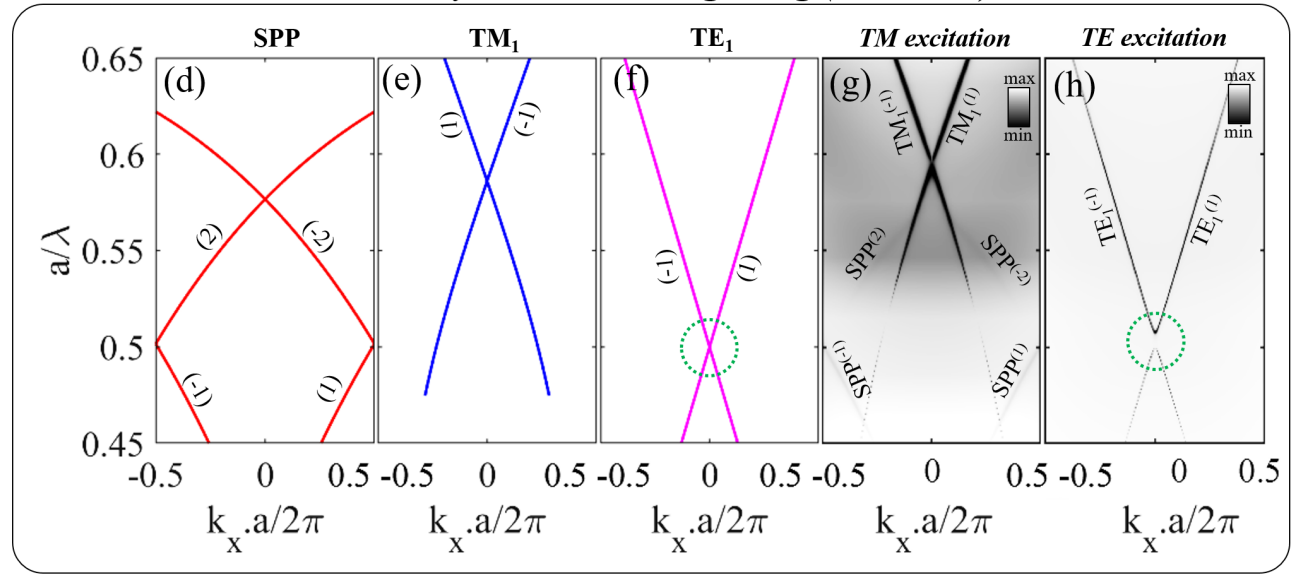

Fig. 2. (a) Design of the perovskite metasurface. (b,d,e,f) Folded dispersion of different guided modes extracted from Figs.1.(b,e,f) with period $a=300 \mathrm{~nm}$. (c,g,h) Angle-resolved reflectivity spectra obtained from RCWA calculations for (c) $h=30 \mathrm{~nm}$ and (g,h) $h=150 \mathrm{~nm}$. All of these calculations are carried out with $a=300 \mathrm{~nm}$, $\eta=0.3, f=0.9$.

tum space with respect to the original guided mode. The dispersion of Bloch resonances are also numerically calculated via angle-resolved reflectivity simulations using Rigourous Coupled Wave Analysis method [64]. The RCWA calculations, shown in Figs.2.(c,g,h), are in good agreement with the band-folding approach. From these results, we identify two pairs of first-order diffraction: $\mathrm{SPP}^{( \pm 1)}$ with $h=30 \mathrm{~nm}$; and $\mathrm{TE}_{1}^{( \pm 1)}$ with $h=150 \mathrm{~nm}$ which undergo strong diffraction efficiency and also are well isolated from other modes, for band-gap engineering. 


\section{Theory of band-gap engineering via diffractive and radiative coupling}

In this section, we will develop a simple theory to describe the band-gap engineering when coupling counter-propagating Bloch resonances. As shown in the previous section, in the vicinity of $k_{x}=0$, the dispersion of counter-propagating resonances $\phi_{ \pm}$is almost linear, thus can be described by:

$$
E_{ \pm}\left(k_{x}\right)=E_{0} \pm \alpha k_{x}
$$

where the slope $\alpha$ represents the group velocity. We assume that the radiative loss $\Gamma_{r}$ via coupling to the radiative continuum, and the non-radiative losses $\Gamma_{n r}$ due to absorption in metal depend weakly on $k_{x}$. The couplings between $\phi_{+}$and $\phi_{-}$consist of direct coupling of strength $U$ due to diffraction mechanism, and also an indirect coupling through radiative losses via the radiative continuum. In the basis of $\left\{\phi_{+}, \phi_{-}\right\}$, the coupling system is described by a non-hermitian Hamiltonian, given by:

$$
H\left(k_{x}\right)=\left[\begin{array}{cc}
E_{+}\left(k_{x}\right) & U \\
U & E_{-}\left(k_{x}\right)
\end{array}\right]-i\left[\begin{array}{cc}
\Gamma_{r}+\Gamma_{n r} & \Gamma_{r} \\
\Gamma_{r} & \Gamma_{r}+\Gamma_{n r}
\end{array}\right]
$$

The two complex eigenvalues of $H\left(k_{x}\right)$ are given by:

$$
\begin{gathered}
\Omega_{\text {bright }}\left(k_{x}\right)=E_{0}-i\left(\Gamma_{r}+\Gamma_{n r}\right)+\left(U-i \Gamma_{r}\right) \sqrt{1+\left(\frac{\alpha k_{x}}{U-i \Gamma_{r}}\right)^{2}} \\
\Omega_{\text {dark }}\left(k_{x}\right)=E_{0}-i\left(\Gamma_{r}+\Gamma_{n r}\right)-\left(U-i \Gamma_{r}\right) \sqrt{1+\left(\frac{\alpha k_{x}}{U-i \Gamma_{r}}\right)^{2}}
\end{gathered}
$$

At $k_{x}=0$, the previous expressions are greatly simplified, and become:

$$
\begin{aligned}
\Omega_{\text {bright }}\left(k_{x}=0\right) & =E_{0}+U-i\left(2 \Gamma_{r}+\Gamma_{n r}\right) \\
\Omega_{\text {dark }}\left(k_{x}=0\right) & =E_{0}-U-i \Gamma_{n r}
\end{aligned}
$$

We obtain therefore a band-gap in the size of $2|U|$ at $k_{x}=0$. Most interestingly, while the two eigenmodes still have the same non-radiative losses $\Gamma_{n r}$, the bright mode takes all radiative losses $2 \Gamma_{r}$ while the dark mode has no radiative losses. Such a feature explains the naming of these modes: only the bright mode can radiate to the free-space. Moreover, if the non-radiative losses is small enough the dark mode will have a very high quality factor, and become a quasi-Bound state In the Continuum (quasi-BIC) [48,49]. Such configuration would be ideal for the strong coupling regime since we would obtain polaritons of very high quality factor.

It is noted that a band-inversion between bright and dark modes occurs when tuning $U$ from positive to negative value, In particular, a very special phenomenon takes place when $U=0$. In this case, the two complex eigenvalues will degenerate:

$$
\Omega_{\text {bright }}=\Omega_{\text {dark }}=E_{0}-i\left(\Gamma_{r}+\Gamma_{n r}\right) \equiv \Omega_{E P}
$$

at

$$
k_{x}= \pm \Gamma_{r} / \alpha \equiv \pm k_{E P}
$$

In non-hermitian physics, such situations are called exceptional points (EPs) [50,51]. The separation $\Delta=2 k_{E P}=2 \Gamma_{r} / \alpha$ between the two EPs in momentum space is driven by the radiative losses $\Gamma_{r}$. Importantly, it is theoretically predicted that the Density Of State (DOS) is greatly enhanced at EPs in periodic lattice [52,65]. As a consequence, using these EPs in a strong coupling regime would greatly enhance relaxation mechanisms to achieve polariton lasing. 


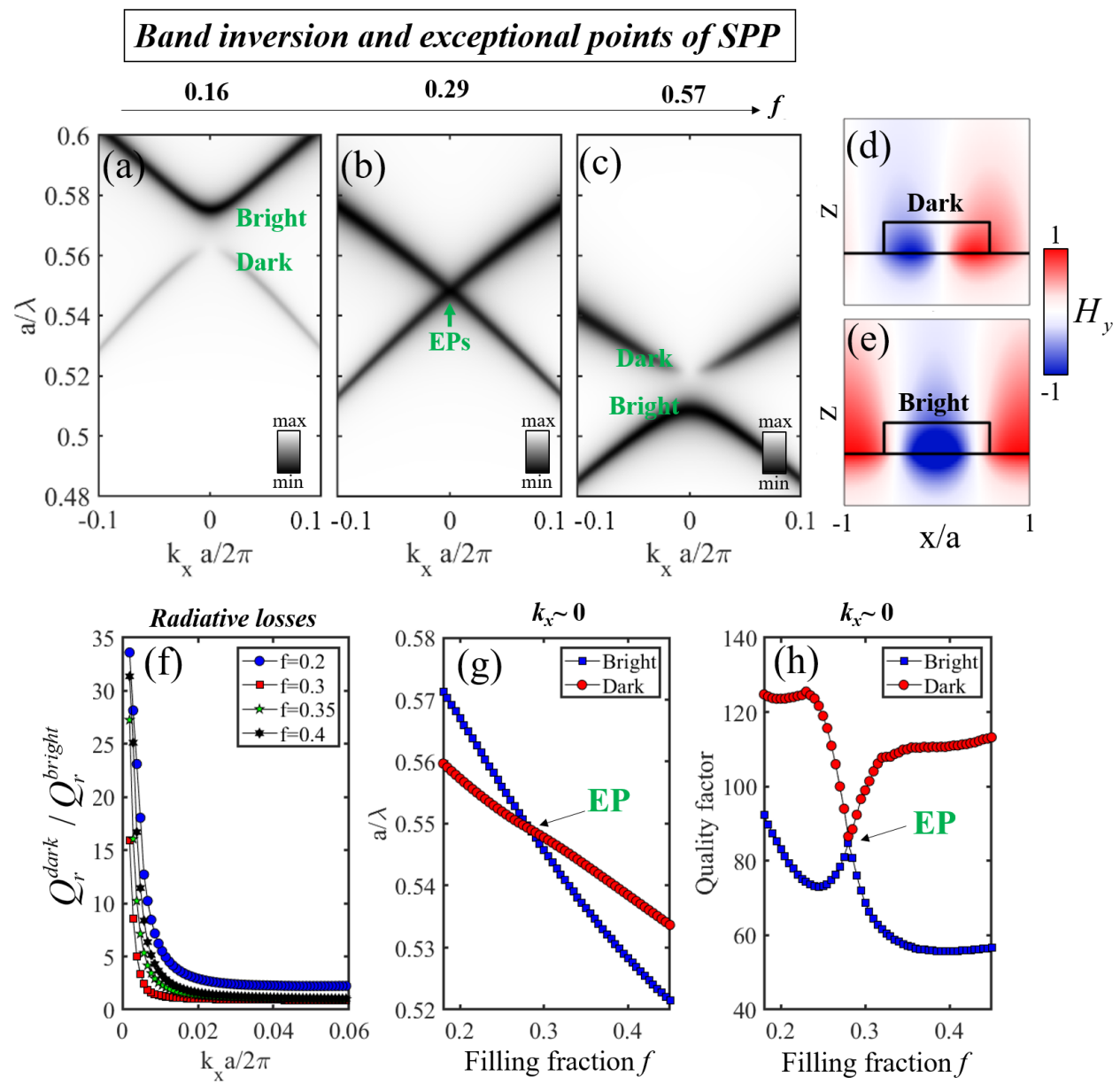

Fig. 3. (a-c) Angle-resolved reflectivity spectra obtained from RCWA calculations for the passive metasurface of (a) $f=0.16$, (b) $f=0.29$, (c) $f=0.57$. All of these calculations are carried out with $a=300 \mathrm{~nm}, \eta=1$ and $h=30 \mathrm{~nm}$. (d,e) Field distribution of the dark and bright modes. (f) Ratio of radiative quality factors as function of $k_{x}$ for different value of the filling fraction $f$. (g,h) Spectral position and quality factor of the dark and bright modes at $k_{x}=0$ as function of the filling fraction $f$.

\section{Band-gap engineering of SPP resonances in ultra-thin metasurface}

We now verify the band-gap engineering theory presented in the previous section with the coupling between $S P P^{( \pm 1)}$ in ultra-thin metasurface $(h=30 \mathrm{~nm})$. The etching factor $\eta$ is fixed at unity while the filling fraction $f$ is the parameter for tuning the diffracting coupling strength $U$ mentioned in the theory. Figures.3.(a-c) present angle-resolved reflectivity spectra obtained via RCWA calculations performed on passive structures with three different values of the filling fraction $f$. In these figures, the dark mode is easily identified via the vanishing of reflectivity dip at $k_{x}=0$. The observed dark(bright) nature is well consistent with the odd(even) parity in $\sigma_{x}$ mirror symmetry of the field distribution [Fig.3.(d,e)]: only symmetric mode can couple to the radiative continuum. The ratio $Q_{r}^{\text {dark }} / Q_{r}^{\text {bright }}$ as function of $k_{x}$ for different filling fraction $f$ is 
depicted in Fig.3.(f). It shows that the $Q_{r}^{\text {dark }} \gg Q_{r}^{\text {bright }}$ when $k_{x}$ approaching 0, thus suggest that $Q_{r}^{\text {dark }} \underset{k_{x} \rightarrow 0}{\longrightarrow} \infty$ as expected. Moreover, the results from Figs.3.(a-c) suggest that $U>0$ for $f<0.29$ and $U<0$ for $f>0.29$. In other words, band inversion takes place when scanning $f$ across 0.29 , and $E P$ configuration occurs at $f=0.29$. For further insights into this observation, the quality factor and energy of dark and bright modes at $k_{x}=0$ as functions of filling fraction $f$ are extracted and presented in Figs.3.(g,h). These results show plainly the band-inversion in the vicinity of $f=0.29$ where $E P s$ take place.

Nevertheless, the dispersion at $f=0.29$ [Fig.3.(b)] does not show double EPs feature $\left(k_{x}= \pm k_{E P}\right)$ and looks very similar to a Dirac dispersion, suggesting that $k_{E P} \sim 0$. Otherwise, the enhancement of DOS at EPs, which is accompanied by an enhancement of resonance dip at $\pm k_{E P}$, is not observed. This can be explained by a dominant role of non-radiative losses: if the absorption overtakes the radiative continuum as the main leaky channel, high quality factor features of the dark state, and the DOS enhancement at EPs will be hindered. An evaluation of non-radiative losses versus radiative one is thus needed. We note that for a given resonance, the quality factor $Q$ is composed of the radiative quality factor $Q_{r}$ and the non-radiative one $Q_{n r}$, given by:

$$
\frac{1}{Q}=\frac{1}{Q_{r}}+\frac{1}{Q_{n r}}
$$

Knowing that $Q_{r}^{\text {dark }}\left(k_{x}=0\right)=\infty$ and $Q^{\text {dark }}\left(k_{x}=0\right) \sim 120$ [Fig.3.g], it leads to $Q_{n r} \sim 120$. Moreover, the results from the previous section [i.e. eq.(8)] suggest that the radiative losses should be evaluated at EP when the losses is well balanced between dark and bright modes. Following this argument, with $Q^{E P} \sim 87$ [Fig.3.(h)], we obtain $Q_{r}^{E P} \sim 316>Q_{n r}$. The non-radiative losses are thus the main leaky channel as suspected. For this reason, although the strong coupling regime between these states and excitons can be achieved in the active structure, we do not consider the active structure for this design.

\section{Band-gap engineering of polaritonic modes in perovskite metasurface}

In this section, the coupling between $T E_{1}^{( \pm 1)}$ in perovskite metasurface of thickness $h=150 \mathrm{~nm}$ will be investigated. Qualitatively, this should be similar to the one of SPP modes discussed in the previous sections: formation of dark, bright bands and EP points at band-inversion are expected. Nevertheless, unlike $S P P^{( \pm 1)}, T E_{1}^{( \pm 1)}$ has almost no field at the perovskite-metal interface, thus promise low non-radiative losses. The etching factor $\eta$ is fixed at 0.3 , corresponding to a patterning depth of $45 \mathrm{~nm}$. We adopt the same strategy as the one discussed in the previous section: the filling fraction $f$ is the parameter for band-gap engineering. However, for each value of $f$, the period $a$ is slightly varied so that the mid-gap is always pinned at $2.35 \mathrm{eV}$. This value corresponds to a detuning of $-50 \mathrm{meV}$ with respect to the energy of excitonic resonance.

We first discuss the results of passive structures [see Figs.4.(a-c)]. Band inversion is observed when scanning $f$ across 0.51 which corresponds to the EP configuration. The dark and bright status are also well confirmed via the parity of field distribution [Figs.4.(d,e)]. Figure 4.f depicts the quality factor of the dark and bright mode as function of $k_{x}$. It shows that $Q^{\text {dark }}\left(k_{x}=0\right) \sim 1500$ which is one order of magnitude higher than the one of SPP from the previous section. We also highlight that $Q^{\text {dark }}\left(k_{x}=0\right) \gg Q^{\text {bright }}\left(k_{x}=0\right)$. The dark mode of this design is therefore a quasi-BIC.

Concerning the EP configuration, its appearance is characterized by an anomaly in the dispersion mapping: the dispersion curve is vertically splitted into two half and separated by a small 


\section{Exceptional point and band inversion of DGM}
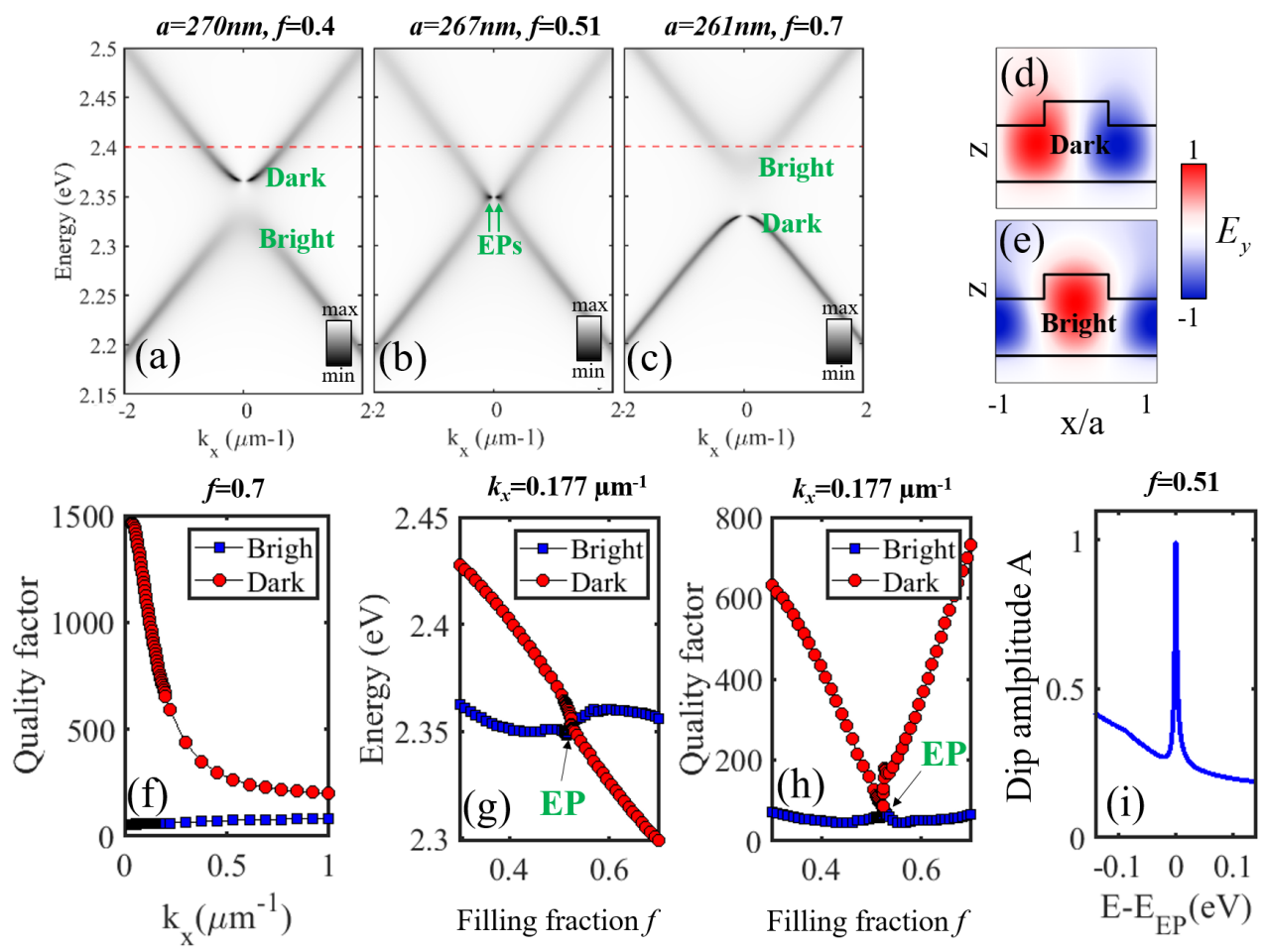

Fig. 4. (a-c) Angle-resolved reflectivity spectra obtained from RCWA calculations for the passive metasurface of (a) $f=0.4$ and $a=270 \mathrm{~nm}$, (b) $f=0.51$ and $a=267 \mathrm{~nm}$, (c) $f=0.7$ and $a=261 \mathrm{~nm}$. All of these calculations are carried out with $\eta=0.3$ and $h=150 \mathrm{~nm}$. (d,e) Field distribution of the dark and bright modes. (f) Quality factor of the bright and dark mode in (c) as function of $k_{x}$. (e,f) Spectral position and quality factor of the dark and bright modes at $k_{x}=k_{E P}=0.177 \mu m^{-1}$ as function of the filling fraction $f$. In these calculations, the period $a$ is fixed at $265 \mathrm{~nm}$. (i) Dip amplitude of the reflectivity resonance as function of the spectral detuning to EP.

"slit" with a minimum at $\mathrm{kx}=0$ corresponding to the gap between both EPs [see Fig.4.(b)] [66]. With this rule, the two EPs can be easily identified at $\pm k_{E P}= \pm 0.177 \mu \mathrm{m}^{-1}$. Indeed, pinning at $k_{x}=0.177 \mu \mathrm{m}^{-1}$, the spectral position and quality factor of the two modes are monitored while scanning $f$. The result [Figs.4.(g,h)] clearly demonstrates the EP configuration at $f=0.51$ with the simultaneous degeneracy of both energy and quality factor. Strikingly, as shown in Fig.4.b, the dip resonance is strongly enhanced at EPs. Figure.4.i presents the dip amplitude as function of its detuning to EP. It indicates that there is a four-fold enhancement of resonance amplitude at EPs. Such observation is perfectly inline with theoretical prediction that the DOS is four-fold enhanced at EPs [52].

Since quasi-BIC and EP features are nicely achieved, the main leaky channel in this design should be the radiative continuum. Indeed, as discussed in the previous section, the non-radiative losses is given by the losses of dark mode at $k_{x}=0: Q_{n r}=Q^{\text {dark }}\left(k_{x}=0\right) \sim 1500$. Moreover, $Q^{E P} \sim 50 \ll Q_{n r}$, that leads to $Q_{r}^{E P} \sim 50 \ll Q_{n r}$. The non-radiative losses is thus negligible. 


\section{Exceptional point and band inversion of exciton-polaritons}
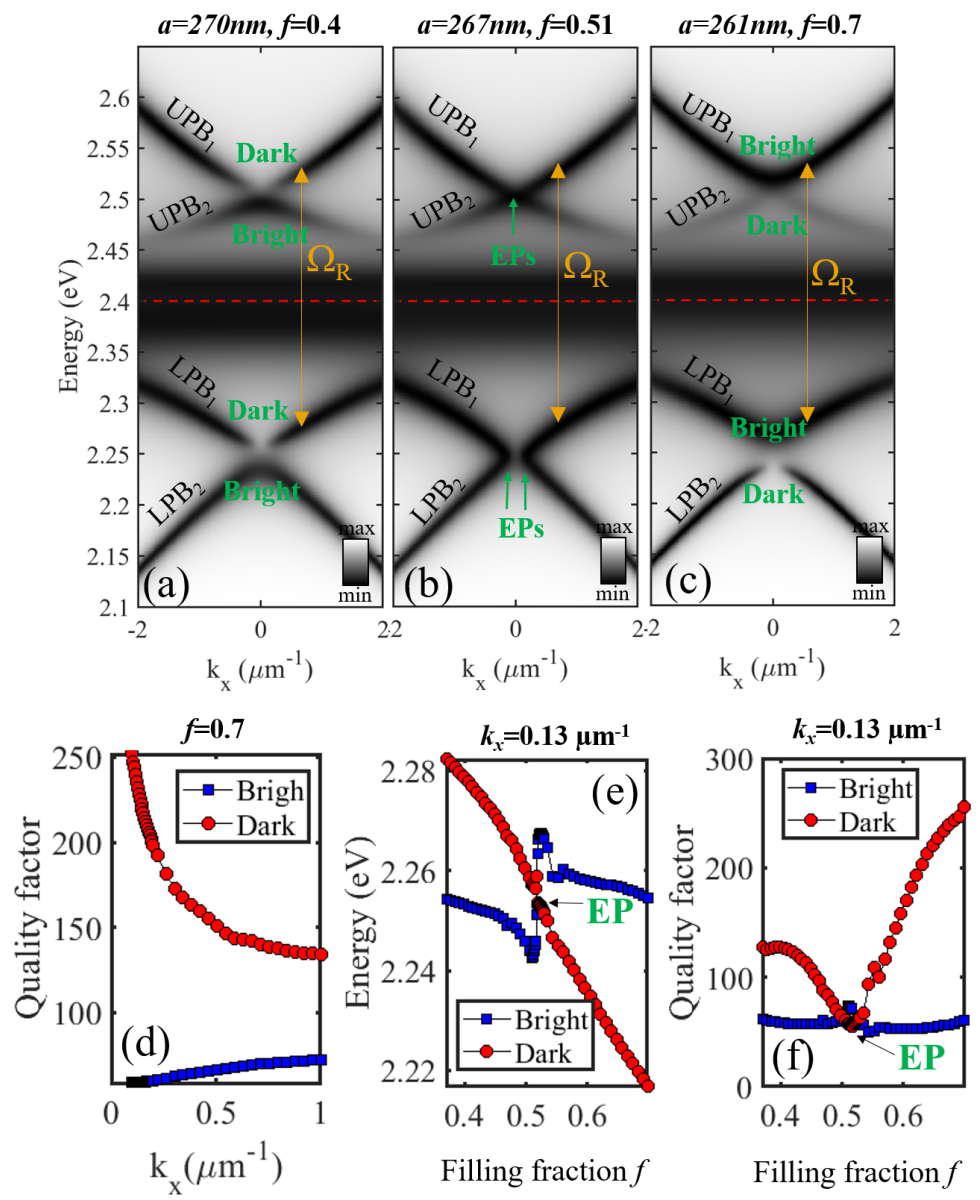

Fig. 5. (a-c) Angle-resolved reflectivity spectra obtained from RCWA calculations for the active metasurface of (a) $f=0.4$ and $a=270 \mathrm{~nm}$, (b) $f=0.51$ and $a=267 \mathrm{~nm}$, (c) $f=0.7$ and $a=261 \mathrm{~nm}$. All of these calculations are carried out with $\eta=0.3$ and $h=150 \mathrm{~nm}$. (d) Quality factor of the dark and bright polaritons in (c) as function of $k_{x}$. (g,h) Spectral position and quality factor of the dark and bright polaritons at $k_{x}=k_{E P}=0.13 \mu \mathrm{m}^{-1}$ as function of the filling fraction $f$. In these calculations, the period $a$ is fixed at $265 \mathrm{~nm}$.

We now study the active structures based on the same designs. As shown in Figs.5.(a-c), four polaritonic modes are observed: two lower and two upper polariton branches. The polaritons states inherit the dark/bright nature of the initial photonic modes with one pair of dark/bright modes on each side of the exciton energy. We highlight that band inversion also takes place at $f=0.51$ as in the case of the passive structure, but occurs for both upper and lower polariton branches. To study rigorously these polaritonic modes, a quantum description of photonic crystal polaritons is required [67]. As a matter of fact, we propose here a simple model to explain their formation. Naively, one could suggest a model of three-coupled oscillators involving the two photonic modes, and the excitonic resonance. However, the field distribution of dark and bright modes [Figs.4.(d,e)] indicate that anti-node position of each mode corresponds to node position 
of the other one. Thus a given exciton in the perovskite metasurface cannot coupled efficiently to both photonic modes. To a first approximation, we can consider that half of excitons only couples to the dark mode, and the other half only couples to the bright mode. With two types excitons and two photonic modes, the system is now described by a four-coupled oscillators Hamiltonian, given by:

$$
H_{p o l}\left(k_{x}\right)=\left[\begin{array}{cccc}
E_{X}-i \Gamma_{X} & V_{\text {bright }} & 0 & 0 \\
V_{\text {bright }} & \Omega_{\text {bright }}\left(k_{x}\right) & 0 & 0 \\
0 & 0 & E_{X}-i \Gamma_{X} & V_{\text {dark }} \\
0 & 0 & V_{\text {dark }} & \Omega_{\text {dark }}\left(k_{x}\right)
\end{array}\right]
$$

The eigenvalues of each $2 \times 2$ bloc of $H_{p o l}$ correspond to a pair upper/lower polariton branches with the same dark or bright nature. Thus we have a set of four polaritonic branches as shown in Figs.5.(a-c). With this model, we can now interpret an anti-crossing effect in each configuration of Figs.5.(a-c). The estimated Rabi splitting amount to $\Omega_{R} \sim 256 \mathrm{meV}$ for anti-crossings of both dark modes [Fig.5.(a)] and bright modes [Fig.5.(c)]. Therefore: $V_{\text {dark }} \sim V_{\text {bright }} \sim \Omega_{R} / 2$. As a consequence, polaritonic modes undergo the same degeneration as the degeneration between original dark and bright photonic modes at $f=0.51$.

The quality factor of lower polariton branches for $f=0.7$ are presented in Fig.5.(d). The narrowing of dark-polariton when $k_{x}$ approaching 0 , together with $Q^{\text {dark-pol }} \gg Q^{\text {bright-pol }}$, clearly indicate that the dark polariton also inherits the quasi-BIC behavior of the original dark mode. We highlight that a similar observation has been recently reported in the framework of strong coupling between excitons in two-dimensional materials and dielectric Bloch resonance [68].

We now focus on the EP configuration at $f=0.51$. Using the criterion mentioned previously with the passive structure, we obtain $k_{E P}^{L P B}=0.13 \mu \mathrm{m}^{-1}$ and $k_{E P}^{U P B} \sim 0$ for EPs of LPBs and UPBs respectively. Indeed, Figs.5.(e,f) depict the spectral position and the quality factor of the dark and bright polaritons of lower branch while keeping $k_{x}=0.13 \mu \mathrm{m}^{-1}$. These data show nicely the creation of polaritonic EP at $f=0.51$. In addition, with a detuning $-50 \mathrm{meV}$ between the photonic EP and the excitonic resonance [Fig.4.(b)] and a Rabi splitting of $256 \mathrm{meV}$, the excitonic and photonic fractions of polaritonic EPs are (32\% exciton, $68 \%$ photon) for EPs of lower branch and (68\% exciton, 32\% photon) for EPs of upper branch. The lower polaritonic EP is therefore more photonic-like while the upper one is more excitonic-like. Since the excitonic losses (given by $\Gamma_{X}$ ) is much more important than the photonic losses (given by $\Gamma_{r}$ and $\Gamma_{n r}$ ), the upper EPs exhibit much more losses than the lower ones. This explains a less pronounced EP feature (i.e. $k_{E P} \sim 0$ ) in the upper branch. Finally, it is important to mention that several groups have reported on polaritonic EPs, but only for localized resonators $[69,70]$. Our results propose the first polaritonic EPs in band structures of momentum space. This promises the entrance of polaritonic physics in non-hermitian topology, in which Dirac points are replaced by EPs as singularities $[71,72]$.

\section{Conclusion}

In conclusion, we have successfully introduced a simple design to obtain polaritonic quasi-BIC and EPs with perovskite plasmonic metasurface. The implementation of such a design can be achieved with current technology of sub-wavelength scale perovskite texturing [41, 47, 56, 58-62, 73-76]. For device development, our results propose an elegant solution to integrate metallic layer into polaritonic devices with minimum photonic degradation. Attractive applications of our design include electrically pumped polariton laser using high-quality factor quasi-BIC or EP with at least 
four-fold enhanced DOS [52,65]. In particular, for fundamental photonic research, this finding would pave the way to use the high nonlinearity of perovskite polaritons to demonstrate many intriguing features of nonlinear BIC such as multistability, self-focusing BIC and breaking of symmetry protected BIC [77,78]. Finally, the possibility to drive dynamically this non-hermitian system around the EP via nonlinearity and electrical field would be a new paradigm for exploring non-hermitian topology [71,72,79].

\section{Funding}

POPEYE (ANR-17-CE24-0020); EMIPERO (ANR-18-CE24-0016); PAI2020 (20 006711 01)

\section{Acknowledgments}

This work is partly supported by the French National Research Agency (ANR) under the project POPEYE (ANR-17-CE24-0020) and project EMIPERO (ANR-18-CE24-0016). It is also supported by the Auvergne-Rhône-Alpes region in the framework of PAI2020.

\section{Disclosures}

"The authors declare no conflicts of interest."

\section{References}

1. C. Weisbuch, M. Nishioka, A. Ishikawa, and Y. Arakawa, "Observation of the coupled exciton-photon mode splitting in a semiconductor quantum microcavity," Phys. Rev. Lett. 69, 3314-3317 (1992).

2. T. K. Hakala, A. J. Moilanen, A. I. Väkeväinen, R. Guo, J.-P. Martikainen, K. S. Daskalakis, H. T. Rekola, A. Julku, and P. Törmä, "Bose-einstein condensation in a plasmonic lattice," Nat. Phys. 14, 739-744 (2018).

3. M. Ramezani, Q. Le-Van, A. Halpin, and J. Gómez Rivas, "Nonlinear emission of molecular ensembles strongly coupled to plasmonic lattices with structural imperfections," Phys. Rev. Lett. 121, 243904 (2018).

4. J. Bellessa, C. Bonnand, J. C. Plenet, and J. Mugnier, "Strong coupling between surface plasmons and excitons in an organic semiconductor," Phys. Rev. Lett. 93, 036404 (2004).

5. J. Dintinger, S. Klein, F. Bustos, W. L. Barnes, and T. W. Ebbesen, "Strong coupling between surface plasmonpolaritons and organic molecules in subwavelength hole arrays," Phys. Rev. B 71, 035424 (2005).

6. C. Symonds, J. Bellessa, J. Plenet, A. Bréhier, R. Parashkov, J.-S. Lauret, and E. Deleporte, "Emission of hybrid organic-inorganic exciton/plasmon mixed states," Appl. Phys. Lett. 90, 091107 (2007).

7. C. Symonds, C. Bonnand, J. Plenet, A. Bréhier, R. Parashkov, J. Lauret, E. Deleporte, and J. Bellessa, "Particularities of surface plasmon-exciton strong coupling with large rabi splitting," New J. Phys. 10, 065017 (2008).

8. F. van Beijnum, P. J. van Veldhoven, E. J. Geluk, M. J. A. de Dood, G. W. 't Hooft, and M. P. van Exter, "Surface plasmon lasing observed in metal hole arrays," Phys. Rev. Lett. 110, 206802 (2013).

9. T. Freixanet, B. Sermage, A. Tiberj, and R. Planel, "In-plane propagation of excitonic cavity polaritons," Phys. Rev. B 61, 7233 (2000).

10. S. A. Guebrou, J. Laverdant, C. Symonds, S. Vignoli, and J. Bellessa, "Spatial coherence properties of surface plasmon investigated by youngs slit experiment," Opt. Lett. 37, 2139-2141 (2012).

11. R. Houdré, C. Weisbuch, R. P. Stanley, U. Oesterle, P. Pellandini, and M. Ilegems, "Measurement of cavity-polariton dispersion curve from angle-resolved photoluminescence experiments," Phys. Rev. Lett. 73, 2043-2046 (1994).

12. M. Wouters, "Resonant polariton-polariton scattering in semiconductor microcavities," Phys. Rev. B 76, 045319 (2007).

13. M. Wouters and I. Carusotto, "Parametric oscillation threshold of semiconductor microcavities in the strong coupling regime," Phys. Rev. B 75, 075332 (2007).

14. T. Byrnes, N. Y. Kim, and Y. Yamamoto, "Exciton-polariton condensates," Nat. Phys. 10, 803-813 (2014).

15. J. Kasprzak, M. Richard, S. Kundermann, A. Baas, P. Jeambrun, J. Keeling, F. Marchetti, M. Szymańska, R. André, J. Staehli et al., "Bose-einstein condensation of exciton polaritons," Nature 443, 409-414 (2006).

16. A. Amo, J. Lefrère, S. Pigeon, C. Adrados, C. Ciuti, I. Carusotto, R. Houdré, E. Giacobino, and A. Bramati, "Superfluidity of polaritons in semiconductor microcavities," Nat. Phys. 5, 805-810 (2009).

17. H. S. Nguyen, D. Gerace, I. Carusotto, D. Sanvitto, E. Galopin, A. Lemaître, I. Sagnes, J. Bloch, and A. Amo, "Acoustic black hole in a stationary hydrodynamic flow of microcavity polaritons," Phys. Rev. Lett. 114, 036402 (2015).

18. P. St-Jean, V. Goblot, E. Galopin, A. Lemaître, T. Ozawa, L. Le Gratiet, I. Sagnes, J. Bloch, and A. Amo, "Lasing in topological edge states of a one-dimensional lattice," Nat. Photonics 11, 651-656 (2017). 
19. S. Klembt, T. H. Harder, O. A. Egorov, K. Winkler, R. Ge, M. A. Bandres, M. Emmerling, L. Worschech, T. C. H. Liew, M. Segev, C. Schneider, and S. Höfling, "Exciton-polariton topological insulator," Nature 562, 552-556 (2018).

20. A. Imamoglu, R. J. Ram, S. Pau, and Y. Yamamoto, "Nonequilibrium condensates and lasers without inversion: Exciton-polariton lasers," Phys. Rev. A 53, 4250-4253 (1996).

21. J. Ciers, J. G. Roch, J.-F. Carlin, G. Jacopin, R. Butté, and N. Grandjean, "Propagating polaritons in iii-nitride slab waveguides," Phys. Rev. Appl. 7, 034019 (2017).

22. O. Jamadi, F. Reveret, P. Disseix, F. Medard, J. Leymarie, A. Moreau, D. Solnyshkov, C. Deparis, M. Leroux, E. Cambril, S. Bouchoule, J. Zuniga-Perez, and G. Malpuech, "Edge-emitting polariton laser and amplifier based on a zno waveguide," Light. Sci. \& Appl. 7, 82 (2018).

23. V. C. Nikolis, A. Mischok, B. Siegmund, J. Kublitski, X. Jia, J. Benduhn, U. Hörmann, D. Neher, M. C. Gather, D. Spoltore, and K. Vandewal, "Strong light-matter coupling for reduced photon energy losses in organic photovoltaics," Nat. Commun. 10, 3706 (2019).

24. Y. Wang, W. Knoll, and J. Dostalek, "Bacterial pathogen surface plasmon resonance biosensor advanced by long range surface plasmons and magnetic nanoparticle assays," Anal. Chem. 84, 8345-8350 (2012).

25. J. A. Hutchison, T. Schwartz, C. Genet, E. Devaux, and T. W. Ebbesen, "Modifying chemical landscapes by coupling to vacuum fields," Angewandte Chemie Int. Ed. 51, 1592-1596 (2012).

26. A. Thomas, J. George, A. Shalabney, M. Dryzhakov, S. J. Varma, J. Moran, T. Chervy, X. Zhong, E. Devaux, C. Genet, J. A. Hutchison, and T. W. Ebbesen, "Ground-state chemical reactivity under vibrational coupling to the vacuum electromagnetic field," Angewandte Chemie Int. Ed. 55, 11462-11466 (2016).

27. K. Abdel-Baki, F. Boitier, H. Diab, G. Lanty, K. Jemli, F. Lédée, D. Garrot, E. Deleporte, and J. S. Lauret, "Exciton dynamics and non-linearities in two-dimensional hybrid organic perovskites," J. Appl. Phys. 119, 1-7 (2016).

28. A. Fieramosca, L. Polimeno, V. Ardizzone, L. De Marco, M. Pugliese, V. Maiorano, M. De Giorgi, L. Dominici, G. Gigli, D. Gerace, D. Ballarini, and D. Sanvitto, "Two-Dimensional hybrid perovskites sustaining strong polariton interactions at room temperature," Sci. Adv. 5, 1-7 (2019).

29. T. Ishihara, "Optical properties of PbI-based perovskite structures," J. Lumin. 60-61, 269-274 (1994).

30. N. Takada, T. Kamata, and D. D. C. Bradley, "Polariton emission from polysilane-based organic microcavities," Appl. Phys. Lett. 82, 1812-1814 (2003).

31. G. Lanty, A. Bréhier, R. Parashkov, J.-S. Lauret, and E. Deleporte, "Strong exciton-photon coupling at room temperature in microcavities containing two-dimensional layered perovskite compounds," New J. Phys. 10, 65007 (2008).

32. R. Su, C. Diederichs, J. Wang, T. C. H. Liew, J. Zhao, S. Liu, W. Xu, Z. Chen, and Q. Xiong, "Room-Temperature Polariton Lasing in All-Inorganic Perovskite Nanoplatelets," Nano Lett. 17, 3982-3988 (2017).

33. R. Su, J. Wang, J. Zhao, J. Xing, W. Zhao, C. Diederichs, T. C. H. Liew, and Q. Xiong, "Room temperature long-range coherent exciton polariton condensate flow in lead halide perovskites," Sci. Adv. 4 (2018).

34. T. J. S. Evans, A. Schlaus, Y. Fu, X. Zhong, T. L. Atallah, M. S. Spencer, L. E. Brus, S. Jin, and X.-Y. Zhu, "Continuous-Wave Lasing in Cesium Lead Bromide Perovskite Nanowires," Adv. Opt. Mater. 6, 1700982 (2018).

35. W. Bao, X. Liu, F. Xue, F. Zheng, R. Tao, S. Wang, Y. Xia, M. Zhao, J. Kim, S. Yang, Q. Li, Y. Wang, Y. Wang, L.-W. Wang, A. H. MacDonald, and X. Zhang, "Observation of Rydberg exciton polaritons and their condensate in a perovskite cavity,” Proc. Natl. Acad. Sci. 116, 20274-20279 (2019).

36. R. Su, S. Ghosh, J. Wang, S. Liu, C. Diederichs, T. C. H. Liew, and Q. Xiong, "Observation of exciton polariton condensation in a perovskite lattice at room temperature," Nat. Phys. (2020).

37. L. Polimeno, A. Fieramosca, G. Lerario, M. Cinquino, M. De Giorgi, D. Ballarini, F. Todisco, L. Dominici, V. Ardizzone, M. Pugliese, C. T. Prontera, V. Maiorano, G. Gigli, L. De Marco, and D. Sanvitto, "Observation of Two Thresholds Leading to Polariton Condensation in 2D Hybrid Perovskites," Adv. Opt. Mater. p. 2000176 (2020).

38. S. Zhang, J. Chen, J. Shi, L. Fu, W. Du, X. Sui, Y. Mi, Z. Jia, F. Liu, J. Shi, X. Wu, N. Tang, Q. Zhang, and X. Liu, "Trapped exciton-polariton condensate by spatial confinement in a perovskite microcavity," ACS Photonics 7, 327-337 (2020).

39. Q. Shang, M. Li, L. Zhao, D. Chen, S. Zhang, S. Chen, P. Gao, C. Shen, J. Xing, G. Xing, B. Shen, X. Liu, and Q. Zhang, "Role of the exciton-polariton in a continuous-wave optically pumped cspbbr3 perovskite laser," Nano Lett. (2020).

40. S.-T. Ha, C. Shen, J. Zhang, and Q. Xiong, "Laser cooling of organic-inorganic lead halide perovskites," Nat. Photonics 10, 115-121 (2016).

41. W. Niu, L. A. Ibbotson, D. Leipold, E. Runge, G. V. Prakash, and J. J. Baumberg, "Image excitons and plasmon-exciton strong coupling in two-dimensional perovskite semiconductors," Phys. Rev. B 91, 161303 (2015).

42. H. S. Nguyen, Z. Han, K. Abdel-Baki, X. Lafosse, A. Amo, J. S. Lauret, E. Deleporte, S. Bouchoule, and J. Bloch, "Quantum confinement of zero-dimensional hybrid organic-inorganic polaritons at room temperature," Appl. Phys. Lett. 104, 1-5 (2014).

43. S. Zhang, Q. Shang, W. Du, J. Shi, Z. Wu, Y. Mi, J. Chen, F. Liu, Y. Li, M. Liu, Q. Zhang, and X. Liu, "Strong Exciton-Photon Coupling in Hybrid Inorganic-Organic Perovskite Micro/Nanowires," Adv. Opt. Mater. 6, 1701032 (2018).

44. Q. Shang, S. Zhang, Z. Liu, J. Chen, P. Yang, C. Li, W. Li, Y. Zhang, Q. Xiong, X. Liu, and Q. Zhang, "Surface Plasmon Enhanced Strong Exciton-Photon Coupling in Hybrid Inorganic-Organic Perovskite Nanowires," Nano Lett. 18, 3335-3343 (2018). 
45. T. Fujita, Y. Sato, T. Kuitani, and T. Ishihara, “Tunable polariton absorption of distributed feedback microcavities at room temperature," Phys. Rev. B 57, 12428-12434 (1998).

46. J. Ishi-Hayase and T. Ishihara, "Fundamental optical properties of photonic crystal slabs in the strong coupling regime," Semicond. Sci. Technol. 18, S411-S418 (2003).

47. N. H. M. Dang, D. Gerace, E. Drouard, G. Trippé-Allard, F. Lédée, R. Mazurczyk, E. Deleporte, C. Seassal, and H. S. Nguyen, "Tailoring dispersion of room-temperature exciton-polaritons with perovskite-based subwavelength metasurfaces," Nano Lett. 20, 2113-2119 (2020).

48. B. Zhen, C. W. Hsu, L. Lu, a. D. Stone, and M. Soljačić, "Topological Nature of Optical Bound States in the Continuum," Phys. Rev. Lett. 113, 1-5 (2014).

49. C. W. Hsu, B. Zhen, A. D. Stone, J. D. Joannopoulos, and M. Soljačić, "Bound states in the continuum,” Nat. Rev. Mater. 1, 16048 (2016).

50. M.-A. Miri and A. Alù, "Exceptional points in optics and photonics," Science 363 (2019).

51. B. Zhen, C. W. Hsu, Y. Igarashi, L. Lu, I. Kaminer, A. Pick, S.-L. Chua, J. D. Joannopoulos, and M. Soljačić, "Spawning rings of exceptional points out of Dirac cones," Nature 525, 354-358 (2015).

52. A. Pick, B. Zhen, O. D. Miller, C. W. Hsu, F. Hernandez, A. W. Rodriguez, M. Soljačić, and S. G. Johnson, "General theory of spontaneous emission near exceptional points," Opt. express 25, 12325-12348 (2017).

53. J.-H. Park, A. Ndao, W. Cai, L. Hsu, A. Kodigala, T. Lepetit, Y.-H. Lo, and B. Kanté, "Symmetry-breaking-induced plasmonic exceptional points and nanoscale sensing," Nat. Phys. 16, 462-468 (2020).

54. S. Zhang, P. Audebert, Y. Wei, A. Al Choueiry, G. Lanty, A. Bréhier, L. Galmiche, G. Clavier, C. Boissière, J.-S. Lauret, and E. Deleporte, "Preparations and Characterizations of Luminescent Two Dimensional Organic-inorganic Perovskite Semiconductors," Materials 3, 3385-3406 (2010).

55. P. B. Johnson and R.-W. Christy, "Optical constants of the noble metals," Phys. review B 6, 4370 (1972).

56. C. Huang, C. Zhang, S. Xiao, Y. Wang, Y. Fan, Y. Liu, N. Zhang, G. Qu, H. Ji, J. Han, L. Ge, Y. Kivshar, and Q. Song, "Ultrafast control of vortex microlasers," Science 367, 1018-1021 (2020).

57. Y. Akimov, M. E. Pam, and S. Sun, "Kretschmann-raether configuration: Revision of the theory of resonant interaction," Phys. Rev. B 96, 155433 (2017).

58. N. Pourdavoud, S. Wang, A. Mayer, T. Hu, Y. Chen, A. Marianovich, W. Kowalsky, R. Heiderhoff, H.-C. Scheer, and T. Riedl, "Photonic nanostructures patterned by thermal nanoimprint directly into organo-metal halide perovskites," Adv. Mater. 29, 1605003 (2017).

59. R. Schmager, G. Gomard, B. S. Richards, and U. W. Paetzold, "Nanophotonic perovskite layers for enhanced current generation and mitigation of lead in perovskite solar cells," Sol. Energy Mater. Sol. Cells 192, 65 - 71 (2019).

60. Z. Li, J. Moon, A. Gharajeh, R. Haroldson, R. Hawkins, W. Hu, A. Zakhidov, and Q. Gu, "Room-temperature continuous-wave operation of organometal halide perovskite lasers," ACS Nano 12, 10968-10976 (2018).

61. Y. Gao, C. Huang, C. Hao, S. Sun, L. Zhang, C. Zhang, Z. Duan, K. Wang, Z. Jin, N. Zhang, A. V. Kildishev, C.-W. Qiu, Q. Song, and S. Xiao, "Lead halide perovskite nanostructures for dynamic color display," ACS Nano 12 8847-8854 (2018).

62. Z. Li, J. S. T. Smalley, R. Haroldson, D. Lin, R. Hawkins, A. Gharajeh, J. Moon, J. Hou, C. Zhang, W. Hu, A. Zakhidov, and Q. Gu, “Active perovskite hyperbolic metasurface,” ACS Photonics 7, 1754-1761 (2020).

63. J. D. Joannopoulos, S. G. Johnson, J. N. Winn, and R. D. Meade, Photonic Crystals: Molding the Flow of Light (Second Edition) (Princeton University Press, 2008), 2nd ed.

64. V. Liu and S. Fan, "S4: A free electromagnetic solver for layered periodic structures," Comput. Phys. Commun. 183, 2233-2244 (2012).

65. Z. Lin, A. Pick, M. Lončar, and A. W. Rodriguez, "Enhanced spontaneous emission at third-order dirac exceptional points in inverse-designed photonic crystals," Phys. Rev. Lett. 117, 107402 (2016).

66. F. Zhong, K. Ding, Y. Zhang, S. Zhu, C. Chan, and H. Liu, "Angle-Resolved Thermal Emission Spectroscopy Characterization of Non-Hermitian Metacrystals," Phys. Rev. Appl. 13, 014071 (2020).

67. D. Gerace and L. C. Andreani, "Quantum theory of exciton-photon coupling in photonic crystal slabs with embedded quantum wells," Phys. Rev. B 75, 235325 (2007).

68. V. Kravtsov, E. Khestanova, F. A. Benimetskiy, T. Ivanova, A. K. Samusev, I. S. Sinev, D. Pidgayko, A. M. Mozharov, I. S. Mukhin, M. S. Lozhkin, Y. V. Kapitonov, A. S. Brichkin, V. D. Kulakovskii, I. A. Shelykh, A. I. Tartakovskii, P. M. Walker, M. S. Skolnick, D. N. Krizhanovskii, and I. V. Iorsh, "Nonlinear polaritons in a monolayer semiconductor coupled to optical bound states in the continuum," Light. Sci. \& Appl. 9, 56 (2020).

69. T. Gao, E. Estrecho, K. Y. Bliokh, T. C. H. Liew, M. D. Fraser, S. Brodbeck, M. Kamp, C. Schneider, S. Höfling, Y. Yamamoto, F. Nori, Y. S. Kivshar, A. G. Truscott, R. G. Dall, and E. A. Ostrovskaya, “Observation of non-Hermitian degeneracies in a chaotic exciton-polariton billiard," Nature 526, 554-558 (2015).

70. T. Gao, G. Li, E. Estrecho, T. C. H. Liew, D. Comber-Todd, A. Nalitov, M. Steger, K. West, L. Pfeiffer, D. W. Snoke, A. V. Kavokin, A. G. Truscott, and E. A. Ostrovskaya, "Chiral modes at exceptional points in exciton-polariton quantum fluids," Phys. Rev. Lett. 120, 065301 (2018).

71. H. Shen, B. Zhen, and L. Fu, “Topological band theory for non-hermitian hamiltonians,” Phys. Rev. Lett. 120, 146402 (2018).

72. K. Kawabata, T. Bessho, and M. Sato, "Classification of exceptional points and non-hermitian topological semimetals," Phys. Rev. Lett. 123, 066405 (2019).

73. M. Saliba, S. M. Wood, J. B. Patel, P. K. Nayak, J. Huang, J. A. Alexander-Webber, B. Wenger, S. D. Stranks, M. T. 
Hörantner, J. T.-W. Wang, R. J. Nicholas, L. M. Herz, M. B. Johnston, S. M. Morris, H. J. Snaith, and M. K. Riede, "Structured organic-inorganic perovskite toward a distributed feedback laser," Adv. Mater. 28, 923-929 (2016).

74. S. Chen, K. Roh, J. Lee, W. K. Chong, Y. Lu, N. Mathews, T. C. Sum, and A. Nurmikko, "A photonic crystal laser from solution based organo-lead iodide perovskite thin films," ACS Nano 10, 3959-3967 (2016).

75. H. Wang, R. Haroldson, B. Balachandran, A. Zakhidov, S. Sohal, J. Y. Chan, A. Zakhidov, and W. Hu, "Nanoimprinted perovskite nanograting photodetector with improved efficiency," ACS Nano 10, 10921-10928 (2016).

76. H. Wang, S.-C. Liu, B. Balachandran, J. Moon, R. Haroldson, Z. Li, A. Ishteev, Q. Gu, W. Zhou, A. Zakhidov, and W. Hu, "Nanoimprinted perovskite metasurface for enhanced photoluminescence," Opt. Express 25, A1162-A1171 (2017).

77. S. D. Krasikov, A. A. Bogdanov, and I. V. Iorsh, "Nonlinear bound states in the continuum of a one-dimensional photonic crystal slab," Phys. Rev. B 97, 224309 (2018).

78. D. N. Maksimov, A. A. Bogdanov, and E. N. Bulgakov, "Optical bistability with bound states in the continuum in dielectric gratings," (2020).

79. L. Feng, R. El-Ganainy, and L. Ge, "Non-Hermitian photonics based on parity-time symmetry," Nat. Photonics 11, 752-762 (2017). 